Acta Crystallographica Section E

Structure Reports

Online

ISSN 1600-5368

\section{1-[3-(4-Chlorophenyl)-6-methyl-1,6-di- hydro-1,2,4,5-tetrazin-1-yl]ethanone}

\section{Feng $\mathrm{Xu}$, Zhenzhen Yang, Junrong Jiang and Lei Shi}

Department of Biological \& Chemical Engineering, Taizhou Vocational \& Technical College, Taizhou, 318000, People's Republic of China

Correspondence e-mail: xufeng901@126.com

Received 18 September 2010; accepted 21 September 2010

Key indicators: single-crystal X-ray study; $T=93 \mathrm{~K}$; mean $\sigma(\mathrm{C}-\mathrm{C})=0.003 \AA$; $R$ factor $=$ $0.049 ; w R$ factor $=0.152 ;$ data-to-parameter ratio $=17.3$.

In the title compound, $\mathrm{C}_{11} \mathrm{H}_{11} \mathrm{ClN}_{4} \mathrm{O}$, the tetrazine ring adopts a non-symmetrical boat conformation. The crystal packing exhibits relatively short intermolecular $\mathrm{C} \cdots \mathrm{N}$ contacts of 3.118 (3) ̊̊.

\section{Related literature}

For related structures, see: Hu et al. (2004, 2005); Jennison et al. (1986); Stam et al. (1982); Xu et al. (2010); Yang et al. (2010). For applications of 1,2,4,5-tetrazine derivatives, see: Sauer (1996).

\section{Experimental}

Crystal data

$\mathrm{C}_{11} \mathrm{H}_{11} \mathrm{ClN}_{4} \mathrm{O}$

$M_{r}=250.69$

Orthorhombic, $\mathrm{Pbca}$

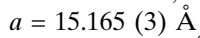

$b=8.0452(15) \AA$

$c=19.349$ (4) $\AA$

$$
\begin{aligned}
& V=2360.7(8) \AA^{3} \\
& Z=8 \\
& \text { Mo } K \alpha \text { radiation } \\
& \mu=0.31 \mathrm{~mm}^{-1} \\
& T=93 \mathrm{~K} \\
& 0.47 \times 0.40 \times 0.37 \mathrm{~mm}
\end{aligned}
$$

\section{Data collection}

Rigaku AFC10/Saturn724+ diffractometer

17617 measured reflections

2705 independent reflections
2599 reflections with $I>2 \sigma(I)$ $R_{\text {int }}=0.038$

\section{Refinement}

$R\left[F^{2}>2 \sigma\left(F^{2}\right)\right]=0.049$

$w R\left(F^{2}\right)=0.152$

$S=1.01$

2705 reflections
156 parameters

$\mathrm{H}$-atom parameters constrained

$\Delta \rho_{\max }=0.28{\mathrm{e} \AA^{-3}}^{-3}$

$\Delta \rho_{\min }=-0.23{\mathrm{e} \AA^{-3}}^{-3}$

Data collection: CrystalClear (Rigaku/MSC, 2008); cell refinement: CrystalClear; data reduction: CrystalClear; program(s) used to solve structure: SHELXS97 (Sheldrick, 2008); program(s) used to refine structure: SHELXL97 (Sheldrick, 2008); molecular graphics: PLATON (Spek, 2009); software used to prepare material for publication: SHELXL97.

We are very grateful to the Science Foundation for the Excellent Youth Scholars of the Department of Education of Zhejiang Province and the Educational Commission of Zhejiang Province of China (grant No. Y201018289).

Supplementary data and figures for this paper are available from the IUCr electronic archives (Reference: CV2768).

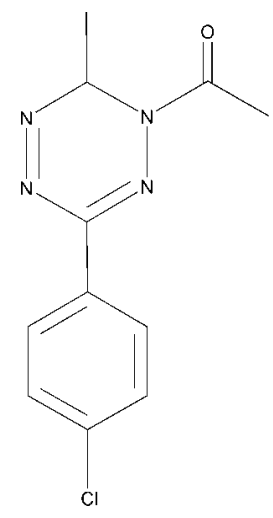

\title{
References
}

Hu, W. X., Rao, G. W. \& Sun, Y. Q. (2004). Bioorg. Med. Chem. Lett. 14, $1177-$ 1181.

Hu, W. X., Shi, H. B., Yuan, Q. \& \&Sun, Y. Q. (2005). J. Chem. Res. pp. 291293.

Jennison, C. P. R., Mackay, D., Watson, K. N. \& Taylor, N. J. (1986). J. Org. Chem. 51, 3043-3051.

Rigaku/MSC (2008). CrystalClear. Rigaku/MSC Inc., The Woodlands, Texas, USA.

Sauer, J. (1996). Comprehensive Heterocyclic Chemistry, 2nd ed., edited by A. J. Boulton, Vol. 6, pp. 901-955. Oxford: Elsevier.

Sheldrick, G. M. (2008). Acta Cryst. A64, 112-122.

Spek, A. L. (2009). Acta Cryst. D65, 148-155.

Stam, C. H., Counotte-Potman, A. D. \& Van der Plas, H. C. (1982). J. Org. Chem. 47, 2856-2858.

Xu, F., Yang, Z. Z., Hu, W. X. \& Xi, L. M. (2010). Chin. J. Org. Chem. 30, 260265.

Yang, Z., Xu, F. \& Chen, H. (2010). Acta Cryst. E66, o969. 


\section{supporting information}

Acta Cryst. (2010). E66, o2651 [doi:10.1107/S1600536810037839]

\section{1-[3-(4-Chlorophenyl)-6-methyl-1,6-dihydro-1,2,4,5-tetrazin-1-yl]ethanone}

\section{Feng Xu, Zhenzhen Yang, Junrong Jiang and Lei Shi}

\section{S1. Comment}

1,2,4,5-Tetrazine derivatives have high potential for biological activity, possessing a wide spectrum of antiviral and antitumor properties. They have been widely used in pesticides and herbicides (Sauer,1996). Dihydro-1,2,4,5- tetrazine has four isomers, namely 1,2-, 1,4-, 1,6- and 3,6-dihydro-1,2,4,5- tetrazines. The 1,6-dihydro structures (Stam et al., 1982; Jennison et al., 1986) were found, by X-ray diffraction, to be homoaromatic. In continuation of our work on the structure-activity relationship of 1,6-dihydro-1,2,4,5-tetrazine derivatives (Hu et al., 2004, 2005), we have obtained the title compound, (I) (Fig.1).

In the tetrazine ring of (I), atoms N1, N2, N3 and N4 are coplanar, while atoms $\mathrm{C} 7$ and $\mathrm{C} 8$ deviate from the plane by 0.254 (3) and 0.621 (3) $\AA$, respectively. The N2/C7/N3 and N1/C8/N4 planes make dihedral angles of $7.61(2)^{\circ}$ and $44.04(2)^{\circ}$, respectively, with the N1/N2/N3/N4 plane, the tetrazine ring adopts an unsymmetrical boat conformation. The benzene ring make dihedral angle of $18.5(2)^{\circ}$ with the N1/N2/N3/N4 plane. $\mathrm{N} 1$ is almost $s p^{2}$ hybridized due to the angles around it add up to $359.9(2)^{\circ}$. Compairing with similar situations in 3-phenyl-6-ethyl- 1,6-dihydro-1,2,4,5-tetrazine (Stam et al., 1982), N-(2-methylphenyl)-3-phenyl-6-methyl-1,6-dihydro-1,2,4,5-tetrazine (Xu et al.,2010), 1-acetyl-3,6dimethyl-1,2,4,5-tetrazine (Jennison et al.,1986) and 1-[3-(4-Methoxyphenyl)-6-methyl-1,6-dihydro-1,2,4,5- tetrazin-1yl]propanone (Yang et al., 2010), one can state that the molecule in (I) is homoaromatic.

\section{S2. Experimental}

3-(4-Chlorophenyl)-6-methyl-1,6-dihydro-1,2,4,5-tetrazine (3.0 mmol), chloroform (10 ml) and pyridine $(0.25 \mathrm{ml}, 3.1$ mmol) were mixed. Acetyl chloride $(3.0 \mathrm{mmol})$ in chloroform $(10 \mathrm{ml})$ was added dropwise with stirring at room temperature. After the starting 1,6-dihydro-1,2,4,5-tetrazine was completely consumed (the reaction courses was monitored by TLC,dichloromethane system), evaporation of the chloroform, crude 1-acetyl-3-(4-chlorophenyl)-6-methyl1,6-dihydro-1,2,4,5-tetrazine was obtained and purified by preparative thin-layer chromatography over silica gel GF254(2 $\mathrm{mm}$ ) (dichloromethane: petroleum ether=1:1). The solution of the compound in anhydrous ethanol was concentrated gradually at room temperature to afford single crystals, which was suitable for X-ray diffraction. (M. P. $352-354 \mathrm{~K}$ ). ${ }^{1} \mathrm{H}$ NMR $\left(\mathrm{CDCl}_{3}\right) \delta$ p.p.m.: $8.10(\mathrm{~d}, 2 \mathrm{H}, \mathrm{J}=8.0 \mathrm{~Hz}), 7.49(\mathrm{~d}, 2 \mathrm{H}, \mathrm{J}=8.0 \mathrm{~Hz}), 6.87(\mathrm{q}, 1 \mathrm{H}, \mathrm{J}=6.7 \mathrm{~Hz}), 2.49(\mathrm{~s}, 3 H), 1.05(\mathrm{~d}, 3 H, \mathrm{~J}$ $=6.4 \mathrm{~Hz})$.

\section{S3. Refinement}

Methyl $\mathrm{H}$ atoms were placed in calculated positions with $\mathrm{C}-\mathrm{H}=0.96 \AA$ and torsion angles were refined to fit the electron density, $U_{\text {iso }}(\mathrm{H})=1.5 U_{\mathrm{eq}}(\mathrm{C})$. Other $\mathrm{H}$ atoms were placed in calculated positions with $\mathrm{C}-\mathrm{H}=0.93$ and $\mathrm{N}-\mathrm{H}=$ $0.86 \AA$, and refined in riding mode, with $U_{\text {iso }}(\mathrm{H})=1.2 U_{\mathrm{eq}}(\mathrm{C}, N)$. 


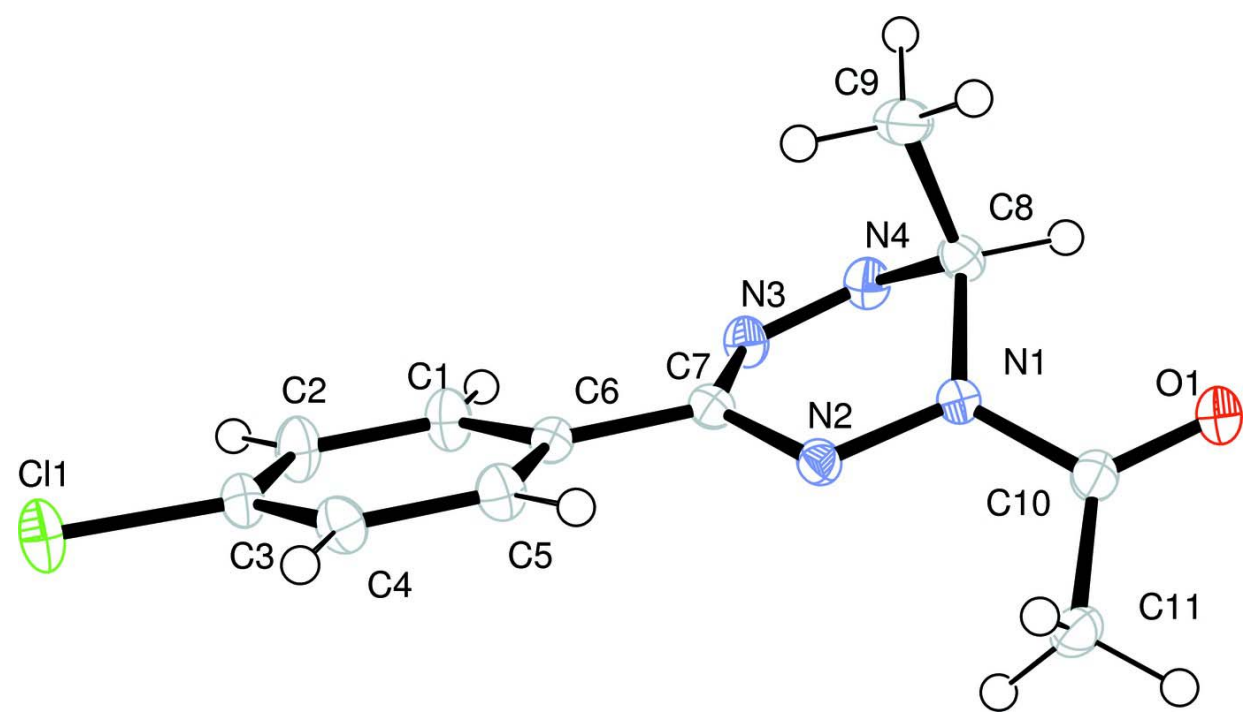

Figure 1

The structure of (I), shown with 30\% probability displacement ellipsoids.

\section{1-[3-(4-Chlorophenyl)-6-methyl-1,6-dihydro-1,2,4,5-tetrazin-1-yl]ethanone}

Crystal data

$\mathrm{C}_{11} \mathrm{H}_{11} \mathrm{ClN}_{4} \mathrm{O}$

$M_{r}=250.69$

Orthorhombic, $\mathrm{Pbca}$

Hall symbol: -P $2 \mathrm{ac} 2 \mathrm{ab}$

$a=15.165(3) \AA$

$b=8.0452(15) \AA$

$c=19.349(4) \AA$

$V=2360.7(8) \AA^{3}$

$Z=8$

\section{Data collection}

Rigaku AFC10/Saturn724+

diffractometer

Radiation source: Rotating Anode

Graphite monochromator

Detector resolution: 28.5714 pixels $\mathrm{mm}^{-1}$

phi and $\omega$ scans

17617 measured reflections

\section{Refinement}

Refinement on $F^{2}$

Least-squares matrix: full

$R\left[F^{2}>2 \sigma\left(F^{2}\right)\right]=0.049$

$w R\left(F^{2}\right)=0.152$

$S=1.01$

2705 reflections

156 parameters

0 restraints

Primary atom site location: structure-invariant direct methods
$F(000)=1040$

$D_{\mathrm{x}}=1.411 \mathrm{Mg} \mathrm{m}^{-3}$

Mo $K \alpha$ radiation, $\lambda=0.71073 \AA$

Cell parameters from 6560 reflections

$\theta=3.1-27.5^{\circ}$

$\mu=0.31 \mathrm{~mm}^{-1}$

$T=93 \mathrm{~K}$

Block, orange

$0.47 \times 0.40 \times 0.37 \mathrm{~mm}$

2705 independent reflections

2599 reflections with $I>2 \sigma(I)$

$R_{\text {int }}=0.038$

$\theta_{\max }=27.5^{\circ}, \theta_{\min }=3.4^{\circ}$

$h=-15 \rightarrow 19$

$k=-7 \rightarrow 10$

$l=-25 \rightarrow 25$

Secondary atom site location: difference Fourier map

Hydrogen site location: inferred from neighbouring sites

$\mathrm{H}$-atom parameters constrained

$w=1 /\left[\sigma^{2}\left(F_{\mathrm{o}}{ }^{2}\right)+(0.1 P)^{2}+1.56 P\right]$

where $P=\left(F_{\mathrm{o}}^{2}+2 F_{\mathrm{c}}{ }^{2}\right) / 3$

$(\Delta / \sigma)_{\max }=0.001$

$\Delta \rho_{\max }=0.28 \mathrm{e} \AA^{-3}$

$\Delta \rho_{\min }=-0.23$ e $\AA^{-3}$ 


\section{Special details}

Geometry. All e.s.d.'s (except the e.s.d. in the dihedral angle between two 1.s. planes) are estimated using the full covariance matrix. The cell e.s.d.'s are taken into account individually in the estimation of e.s.d.'s in distances, angles and torsion angles; correlations between e.s.d.'s in cell parameters are only used when they are defined by crystal symmetry. An approximate (isotropic) treatment of cell e.s.d.'s is used for estimating e.s.d.'s involving 1.s. planes.

Refinement. Refinement of $F^{2}$ against ALL reflections. The weighted $R$-factor $w R$ and goodness of fit $S$ are based on $F^{2}$, conventional $R$-factors $R$ are based on $F$, with $F$ set to zero for negative $F^{2}$. The threshold expression of $F^{2}>\sigma\left(F^{2}\right)$ is used only for calculating $R$-factors (gt) etc. and is not relevant to the choice of reflections for refinement. $R$-factors based on $F^{2}$ are statistically about twice as large as those based on $F$, and $R$ - factors based on ALL data will be even larger.

Fractional atomic coordinates and isotropic or equivalent isotropic displacement parameters $\left(\hat{A}^{2}\right)$

\begin{tabular}{lllll}
\hline & $x$ & $y$ & $z$ & $U_{\text {iso }} / U_{\text {eq }}$ \\
\hline C11 & $0.92289(3)$ & $0.63612(7)$ & $0.27240(2)$ & $0.02638(19)$ \\
O1 & $0.28242(9)$ & $0.51349(19)$ & $0.42261(8)$ & $0.0260(4)$ \\
N1 & $0.42522(10)$ & $0.5869(2)$ & $0.41004(8)$ & $0.0165(3)$ \\
N2 & $0.50513(10)$ & $0.5506(2)$ & $0.38219(8)$ & $0.0167(3)$ \\
N3 & $0.56159(11)$ & $0.7128(2)$ & $0.47435(8)$ & $0.0191(4)$ \\
N4 & $0.48695(11)$ & $0.7527(2)$ & $0.49690(9)$ & $0.0203(4)$ \\
C1 & $0.73156(13)$ & $0.6887(3)$ & $0.41229(10)$ & $0.0226(4)$ \\
H1 & 0.7261 & 0.7262 & 0.4586 & $0.027^{*}$ \\
C2 & $0.81377(13)$ & $0.6872(3)$ & $0.38052(10)$ & $0.0234(4)$ \\
H2 & 0.8647 & 0.7229 & 0.4050 & $0.028^{*}$ \\
C3 & $0.82041(13)$ & $0.6332(2)$ & $0.31290(10)$ & $0.0194(4)$ \\
C4 & $0.74733(13)$ & $0.5800(3)$ & $0.27600(9)$ & $0.0202(4)$ \\
H4 & 0.7531 & 0.5428 & 0.2296 & $0.024^{*}$ \\
C5 & $0.66569(13)$ & $0.5822(3)$ & $0.30804(10)$ & $0.0200(4)$ \\
H5 & 0.6149 & 0.5472 & 0.2832 & $0.024^{*}$ \\
C6 & $0.65710(12)$ & $0.6353(2)$ & $0.37632(10)$ & $0.0168(4)$ \\
C7 & $0.56927(13)$ & $0.6386(2)$ & $0.40816(10)$ & $0.0164(4)$ \\
C8 & $0.41462(13)$ & $0.7456(2)$ & $0.44422(10)$ & $0.0184(4)$ \\
H8 & 0.3564 & 0.7475 & 0.4685 & $0.022^{*}$ \\
C9 & $0.42014(14)$ & $0.8936(3)$ & $0.39576(12)$ & $0.0251(5)$ \\
H9A & 0.4757 & 0.8890 & 0.3699 & $0.030^{*}$ \\
H9B & 0.4179 & 0.9966 & 0.4227 & $0.030^{*}$ \\
H9C & 0.3705 & 0.8906 & 0.3634 & $0.030^{*}$ \\
C10 & $0.35516(13)$ & $0.4761(2)$ & $0.40099(10)$ & $0.0192(4)$ \\
C11 & $0.37533(13)$ & $0.3182(2)$ & $0.36325(11)$ & $0.0217(4)$ \\
H11A & 0.3268 & 0.2393 & 0.3697 & $0.026^{*}$ \\
H11B & 0.4300 & 0.2700 & 0.3814 & $0.026^{*}$ \\
H11C & 0.3825 & 0.3418 & 0.3139 & $0.026^{*}$ \\
& & & & \\
\hline
\end{tabular}

Atomic displacement parameters $\left(\AA^{2}\right)$

\begin{tabular}{lllllll}
\hline & $U^{11}$ & $U^{22}$ & $U^{33}$ & $U^{12}$ & $U^{13}$ & $U^{23}$ \\
\hline C11 & $0.0169(3)$ & $0.0416(4)$ & $0.0206(3)$ & $-0.00189(19)$ & $0.00381(16)$ & $-0.00357(19)$ \\
O1 & $0.0156(7)$ & $0.0260(8)$ & $0.0363(8)$ & $-0.0012(6)$ & $0.0001(6)$ & $0.0006(6)$ \\
N1 & $0.0153(8)$ & $0.0168(8)$ & $0.0175(8)$ & $0.0002(6)$ & $0.0009(6)$ & $0.0000(6)$ \\
N2 & $0.0153(8)$ & $0.0174(8)$ & $0.0174(8)$ & $0.0010(6)$ & $0.0005(6)$ & $0.0019(6)$
\end{tabular}




$\begin{array}{lllllll}\mathrm{N} 3 & 0.0181(8) & 0.0232(9) & 0.0158(8) & -0.0004(6) & 0.0016(6) & -0.0013(6) \\ \mathrm{N} 4 & 0.0195(8) & 0.0231(8) & 0.0184(8) & -0.0015(7) & 0.0008(6) & -0.0024(6) \\ \text { C1 } & 0.0195(10) & 0.0334(11) & 0.0148(8) & -0.0025(8) & 0.0011(7) & -0.0034(8) \\ \text { C2 } & 0.0161(9) & 0.0357(12) & 0.0186(9) & -0.0050(8) & -0.0018(7) & -0.0049(8) \\ \text { C3 } & 0.0158(9) & 0.0237(10) & 0.0186(9) & 0.0000(7) & 0.0024(7) & 0.0008(7) \\ \text { C4 } & 0.0200(10) & 0.0251(10) & 0.0153(8) & 0.0012(8) & 0.0005(7) & -0.0035(7) \\ \text { C5 } & 0.0178(9) & 0.0247(10) & 0.0175(9) & -0.0008(8) & -0.0015(7) & -0.0027(7) \\ \text { C6 } & 0.0163(9) & 0.0170(9) & 0.0172(9) & -0.0010(7) & 0.0002(7) & 0.0005(6) \\ \text { C7 } & 0.0170(10) & 0.0172(9) & 0.0150(9) & 0.0015(7) & -0.0008(6) & 0.0004(6) \\ \text { C8 } & 0.0178(9) & 0.0191(9) & 0.0184(9) & 0.0024(7) & 0.0010(7) & -0.0025(7) \\ \text { C9 } & 0.0264(11) & 0.0180(10) & 0.0309(11) & 0.0030(8) & -0.0019(8) & 0.0014(8) \\ \text { C10 } & 0.0174(9) & 0.0205(10) & 0.0198(9) & -0.0011(7) & -0.0027(7) & 0.0042(7) \\ \text { C11 } & 0.0208(10) & 0.0182(9) & 0.0263(10) & -0.0036(8) & -0.0034(7) & 0.0007(7) \\ \end{array}$

Geometric parameters $\left(\AA,{ }^{\circ}\right)$

\begin{tabular}{|c|c|c|c|}
\hline $\mathrm{C} 11-\mathrm{C} 3$ & $1.741(2)$ & $\mathrm{C} 4-\mathrm{C} 5$ & $1.385(3)$ \\
\hline $\mathrm{O} 1-\mathrm{C} 10$ & $1.218(2)$ & $\mathrm{C} 4-\mathrm{H} 4$ & 0.9500 \\
\hline $\mathrm{N} 1-\mathrm{N} 2$ & $1.358(2)$ & $\mathrm{C} 5-\mathrm{C} 6$ & $1.395(3)$ \\
\hline $\mathrm{N} 1-\mathrm{C} 10$ & $1.398(3)$ & $\mathrm{C} 5-\mathrm{H} 5$ & 0.9500 \\
\hline $\mathrm{N} 1-\mathrm{C} 8$ & $1.447(2)$ & $\mathrm{C} 6-\mathrm{C} 7$ & $1.468(3)$ \\
\hline $\mathrm{N} 2-\mathrm{C} 7$ & $1.304(2)$ & $\mathrm{C} 8-\mathrm{C} 9$ & $1.518(3)$ \\
\hline N3-N4 & $1.255(2)$ & $\mathrm{C} 8-\mathrm{H} 8$ & 1.0000 \\
\hline $\mathrm{N} 3-\mathrm{C} 7$ & $1.418(2)$ & $\mathrm{C} 9-\mathrm{H} 9 \mathrm{~A}$ & 0.9800 \\
\hline $\mathrm{N} 4-\mathrm{C} 8$ & $1.499(2)$ & C9-H9B & 0.9800 \\
\hline $\mathrm{C} 1-\mathrm{C} 2$ & $1.390(3)$ & $\mathrm{C} 9-\mathrm{H} 9 \mathrm{C}$ & 0.9800 \\
\hline $\mathrm{C} 1-\mathrm{C} 6$ & $1.394(3)$ & $\mathrm{C} 10-\mathrm{C} 11$ & $1.497(3)$ \\
\hline $\mathrm{C} 1-\mathrm{H} 1$ & 0.9500 & C11-H11A & 0.9800 \\
\hline $\mathrm{C} 2-\mathrm{C} 3$ & $1.382(3)$ & C11-H11B & 0.9800 \\
\hline $\mathrm{C} 2-\mathrm{H} 2$ & 0.9500 & $\mathrm{C} 11-\mathrm{H} 11 \mathrm{C}$ & 0.9800 \\
\hline $\mathrm{C} 3-\mathrm{C} 4$ & $1.386(3)$ & & \\
\hline $\mathrm{N} 2-\mathrm{N} 1-\mathrm{C} 10$ & $119.42(16)$ & $\mathrm{N} 2-\mathrm{C} 7-\mathrm{N} 3$ & $121.02(17)$ \\
\hline $\mathrm{N} 2-\mathrm{N} 1-\mathrm{C} 8$ & $118.06(15)$ & $\mathrm{N} 2-\mathrm{C} 7-\mathrm{C} 6$ & $120.36(17)$ \\
\hline $\mathrm{C} 10-\mathrm{N} 1-\mathrm{C} 8$ & $122.38(16)$ & $\mathrm{N} 3-\mathrm{C} 7-\mathrm{C} 6$ & $117.48(16)$ \\
\hline $\mathrm{C} 7-\mathrm{N} 2-\mathrm{N} 1$ & $113.33(16)$ & $\mathrm{N} 1-\mathrm{C} 8-\mathrm{N} 4$ & $105.26(15)$ \\
\hline $\mathrm{N} 4-\mathrm{N} 3-\mathrm{C} 7$ & $119.73(16)$ & $\mathrm{N} 1-\mathrm{C} 8-\mathrm{C} 9$ & $113.80(16)$ \\
\hline $\mathrm{N} 3-\mathrm{N} 4-\mathrm{C} 8$ & $114.46(16)$ & $\mathrm{N} 4-\mathrm{C} 8-\mathrm{C} 9$ & $110.49(16)$ \\
\hline $\mathrm{C} 2-\mathrm{C} 1-\mathrm{C} 6$ & $120.20(17)$ & $\mathrm{N} 1-\mathrm{C} 8-\mathrm{H} 8$ & 109.1 \\
\hline $\mathrm{C} 2-\mathrm{C} 1-\mathrm{H} 1$ & 119.9 & $\mathrm{~N} 4-\mathrm{C} 8-\mathrm{H} 8$ & 109.1 \\
\hline $\mathrm{C} 6-\mathrm{C} 1-\mathrm{H} 1$ & 119.9 & $\mathrm{C} 9-\mathrm{C} 8-\mathrm{H} 8$ & 109.1 \\
\hline $\mathrm{C} 3-\mathrm{C} 2-\mathrm{C} 1$ & $119.11(18)$ & $\mathrm{C} 8-\mathrm{C} 9-\mathrm{H} 9 \mathrm{~A}$ & 109.5 \\
\hline $\mathrm{C} 3-\mathrm{C} 2-\mathrm{H} 2$ & 120.4 & $\mathrm{C} 8-\mathrm{C} 9-\mathrm{H} 9 \mathrm{~B}$ & 109.5 \\
\hline $\mathrm{C} 1-\mathrm{C} 2-\mathrm{H} 2$ & 120.4 & $\mathrm{H} 9 \mathrm{~A}-\mathrm{C} 9-\mathrm{H} 9 \mathrm{~B}$ & 109.5 \\
\hline $\mathrm{C} 2-\mathrm{C} 3-\mathrm{C} 4$ & $121.77(18)$ & $\mathrm{C} 8-\mathrm{C} 9-\mathrm{H} 9 \mathrm{C}$ & 109.5 \\
\hline $\mathrm{C} 2-\mathrm{C} 3-\mathrm{C} 11$ & $119.14(15)$ & $\mathrm{H} 9 \mathrm{~A}-\mathrm{C} 9-\mathrm{H} 9 \mathrm{C}$ & 109.5 \\
\hline $\mathrm{C} 4-\mathrm{C} 3-\mathrm{Cl}$ & $119.08(15)$ & $\mathrm{H} 9 \mathrm{~B}-\mathrm{C} 9-\mathrm{H} 9 \mathrm{C}$ & 109.5 \\
\hline $\mathrm{C} 5-\mathrm{C} 4-\mathrm{C} 3$ & $118.70(17)$ & $\mathrm{O} 1-\mathrm{C} 10-\mathrm{N} 1$ & $119.20(18)$ \\
\hline
\end{tabular}




\begin{tabular}{|c|c|c|c|}
\hline $\mathrm{C} 5-\mathrm{C} 4-\mathrm{H} 4$ & 120.7 & $\mathrm{O} 1-\mathrm{C} 10-\mathrm{C} 11$ & $124.23(18)$ \\
\hline $\mathrm{C} 3-\mathrm{C} 4-\mathrm{H} 4$ & 120.7 & $\mathrm{~N} 1-\mathrm{C} 10-\mathrm{C} 11$ & $116.56(17)$ \\
\hline $\mathrm{C} 4-\mathrm{C} 5-\mathrm{C} 6$ & $120.77(18)$ & $\mathrm{C} 10-\mathrm{C} 11-\mathrm{H} 11 \mathrm{~A}$ & 109.5 \\
\hline $\mathrm{C} 4-\mathrm{C} 5-\mathrm{H} 5$ & 119.6 & $\mathrm{C} 10-\mathrm{C} 11-\mathrm{H} 11 \mathrm{~B}$ & 109.5 \\
\hline $\mathrm{C} 6-\mathrm{C} 5-\mathrm{H} 5$ & 119.6 & $\mathrm{H} 11 \mathrm{~A}-\mathrm{C} 11-\mathrm{H} 11 \mathrm{~B}$ & 109.5 \\
\hline $\mathrm{C} 1-\mathrm{C} 6-\mathrm{C} 5$ & $119.45(17)$ & $\mathrm{C} 10-\mathrm{C} 11-\mathrm{H} 11 \mathrm{C}$ & 109.5 \\
\hline $\mathrm{C} 1-\mathrm{C} 6-\mathrm{C} 7$ & $121.32(17)$ & $\mathrm{H} 11 \mathrm{~A}-\mathrm{C} 11-\mathrm{H} 11 \mathrm{C}$ & 109.5 \\
\hline $\mathrm{C} 5-\mathrm{C} 6-\mathrm{C} 7$ & $119.21(17)$ & $\mathrm{H} 11 \mathrm{~B}-\mathrm{C} 11-\mathrm{H} 11 \mathrm{C}$ & 109.5 \\
\hline $\mathrm{C} 10-\mathrm{N} 1-\mathrm{N} 2-\mathrm{C} 7$ & $-163.54(16)$ & $\mathrm{N} 4-\mathrm{N} 3-\mathrm{C} 7-\mathrm{C} 6$ & $163.93(18)$ \\
\hline $\mathrm{C} 8-\mathrm{N} 1-\mathrm{N} 2-\mathrm{C} 7$ & $20.6(2)$ & $\mathrm{C} 1-\mathrm{C} 6-\mathrm{C} 7-\mathrm{N} 2$ & $-162.04(18)$ \\
\hline $\mathrm{C} 7-\mathrm{N} 3-\mathrm{N} 4-\mathrm{C} 8$ & $-11.1(3)$ & $\mathrm{C} 5-\mathrm{C} 6-\mathrm{C} 7-\mathrm{N} 2$ & $19.9(3)$ \\
\hline $\mathrm{C} 6-\mathrm{C} 1-\mathrm{C} 2-\mathrm{C} 3$ & $0.4(3)$ & $\mathrm{C} 1-\mathrm{C} 6-\mathrm{C} 7-\mathrm{N} 3$ & $5.9(3)$ \\
\hline $\mathrm{C} 1-\mathrm{C} 2-\mathrm{C} 3-\mathrm{C} 4$ & $-0.2(3)$ & $\mathrm{C} 5-\mathrm{C} 6-\mathrm{C} 7-\mathrm{N} 3$ & $-172.22(17)$ \\
\hline $\mathrm{C} 1-\mathrm{C} 2-\mathrm{C} 3-\mathrm{Cl1}$ & $178.44(17)$ & $\mathrm{N} 2-\mathrm{N} 1-\mathrm{C} 8-\mathrm{N} 4$ & $-54.1(2)$ \\
\hline $\mathrm{C} 2-\mathrm{C} 3-\mathrm{C} 4-\mathrm{C} 5$ & $0.3(3)$ & $\mathrm{C} 10-\mathrm{N} 1-\mathrm{C} 8-\mathrm{N} 4$ & $130.11(17)$ \\
\hline $\mathrm{C} 11-\mathrm{C} 3-\mathrm{C} 4-\mathrm{C} 5$ & $-178.33(16)$ & $\mathrm{N} 2-\mathrm{N} 1-\mathrm{C} 8-\mathrm{C} 9$ & $67.0(2)$ \\
\hline $\mathrm{C} 3-\mathrm{C} 4-\mathrm{C} 5-\mathrm{C} 6$ & $-0.6(3)$ & $\mathrm{C} 10-\mathrm{N} 1-\mathrm{C} 8-\mathrm{C} 9$ & $-108.8(2)$ \\
\hline $\mathrm{C} 2-\mathrm{C} 1-\mathrm{C} 6-\mathrm{C} 5$ & $-0.7(3)$ & $\mathrm{N} 3-\mathrm{N} 4-\mathrm{C} 8-\mathrm{N} 1$ & $47.4(2)$ \\
\hline $\mathrm{C} 2-\mathrm{C} 1-\mathrm{C} 6-\mathrm{C} 7$ & $-178.79(19)$ & $\mathrm{N} 3-\mathrm{N} 4-\mathrm{C} 8-\mathrm{C} 9$ & $-75.8(2)$ \\
\hline $\mathrm{C} 4-\mathrm{C} 5-\mathrm{C} 6-\mathrm{C} 1$ & $0.8(3)$ & $\mathrm{N} 2-\mathrm{N} 1-\mathrm{C} 10-\mathrm{O} 1$ & $-175.83(17)$ \\
\hline $\mathrm{C} 4-\mathrm{C} 5-\mathrm{C} 6-\mathrm{C} 7$ & $178.96(19)$ & $\mathrm{C} 8-\mathrm{N} 1-\mathrm{C} 10-\mathrm{O} 1$ & $-0.1(3)$ \\
\hline $\mathrm{N} 1-\mathrm{N} 2-\mathrm{C} 7-\mathrm{N} 3$ & $22.6(2)$ & $\mathrm{N} 2-\mathrm{N} 1-\mathrm{C} 10-\mathrm{C} 11$ & $2.9(2)$ \\
\hline $\mathrm{N} 1-\mathrm{N} 2-\mathrm{C} 7-\mathrm{C} 6$ & $-169.92(16)$ & $\mathrm{C} 8-\mathrm{N} 1-\mathrm{C} 10-\mathrm{C} 11$ & $178.63(17)$ \\
\hline $\mathrm{N} 4-\mathrm{N} 3-\mathrm{C} 7-\mathrm{N} 2$ & $-28.2(3)$ & & \\
\hline
\end{tabular}

